\title{
Built-In Potentials Induced by Molecular Order in Amorphous Organic Thin Films
}

\author{
Pascal Friederich, ${ }^{\dagger}$ Vadim Rodin, ${ }^{\ddagger}$ Florian von Wrochem, ${ }^{*} \ddagger \odot$ and Wolfgang Wenzel ${ }^{* \dagger}$ \\ ${ }^{\dagger}$ Institute of Nanotechnology, Karlsruhe Institute of Technology, 76131 Karlsruhe, Germany \\ ${ }^{\ddagger}$ Sony Europe Ltd., Zn Deutschland, 70327 Stuttgart, Germany
}

Supporting Information

\begin{abstract}
Many molecules used to fabricate organic semiconductor devices carry an intrinsic dipole moment. Anisotropic orientation of such molecules in amorphous organic thin films during the deposition process can lead to the spontaneous buildup of an electrostatic potential perpendicular to the film. This so called giant surface potential (GSP) effect can be exploited in organic electronics applications and was extensively studied in experiment. However, presently, an understanding of the molecular mechanism driving the orientation is lacking. Here, we model the physical vapor

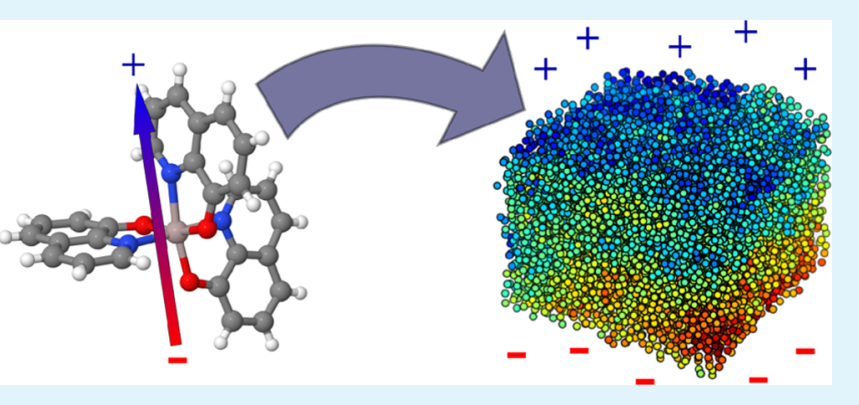
deposition process of seven small organic molecules employed in organic light emitting diode applications with atomistic simulations. We are able to reproduce experimental results for a wide range of strength of the GSP effect. We find that the electrostatic interaction between the dipole moments of the molecules limits the GSP strength and identify short range van der Waals interactions between the molecule and the surface during deposition as the driving force behind the anisotropic orientation. We furthermore show how the GSP effect influences the energy levels responsible for charge transport, which is important for the design of organic semiconductors and devices.
\end{abstract}

KEYWORDS: organic electronics, giant surface potential effect, organic light emitting diodes, simulation of organic semiconductors, built in potential

\section{INTRODUCTION}

One of the major surprises in organic electronics in recent years has been the discovery of the anisotropic orientation of molecules in otherwise amorphous thin films. ${ }^{1-3}$ This phenomenon only occurs in films that are produced using vapor deposition techniques, ${ }^{4}$ whereas solution processed thin films do not show anisotropic orientations of molecules. The preferential in plane orientation of the transition dipole moments of dye molecules is for example used to increase the fraction of photons emitted orthogonal to the substrate. ${ }^{4}$ Several research groups furthermore showed that in pristine organic materials a net orientation of the molecules can lead to a spontaneous buildup of a potential drop within the organic layer. $^{2,3,5}$ The origin of this giant surface potential (GSP) effect lies in the anisotropy in the orientation of molecular dipoles within molecular layer, formed upon vapor deposition of small molecules on inorganic or organic substrates. ${ }^{3}$ In contrast to the limited interface dipole strength formed by thin inorganic coatings (e.g., oxides), adsorbates, or self assembled mono layers, ${ }^{6-8}$ the giant surface potential in thick organic layers can easily reach up to $28 \mathrm{~V}$ for a $500 \mathrm{~nm}$ thick $\mathrm{Alq}_{3}$ layer. ${ }^{3}$ Such a remarkable voltage drop across a thin organic layer has significant implications for organic electronics applications. Furthermore, it was shown that the anisotropic orientation of diluted dye molecules in a matrix of an amorphous host material can be used to enhance the outcoupling efficiency of organic light emitting diodes (OLEDs). ${ }^{9-12}$

The mechanism leading to an anisotropic orientation of molecules is presently actively debated. ${ }^{9,13-18}$ The driving force governing the molecular orientation is believed to be the result of nonbonded interactions, such as electrostatic interaction or van der Waals forces, between molecules and the substrate, but the exact mechanism responsible for the orientation is still unclear. Predictive simulation techniques supply complemen tary information to experiments and help to identify the molecular mechanism by which the anisotropic orientation of small molecules on amorphous substrates takes place.

On the basis of an atomistic Monte Carlo (MC) model that mimics the molecular vapor deposition process on surfaces, we demonstrate here that the giant surface potential effect can be predicted solely using the molecular structure and dipole moment of each of the molecular thin film components as an input. We perform simulations of seven organic molecules, whose GSP was previously assessed experimentally, ${ }^{2}$ and reproduce the correct trends in experimental surface potential values. We furthermore analyze the molecular driving force for 


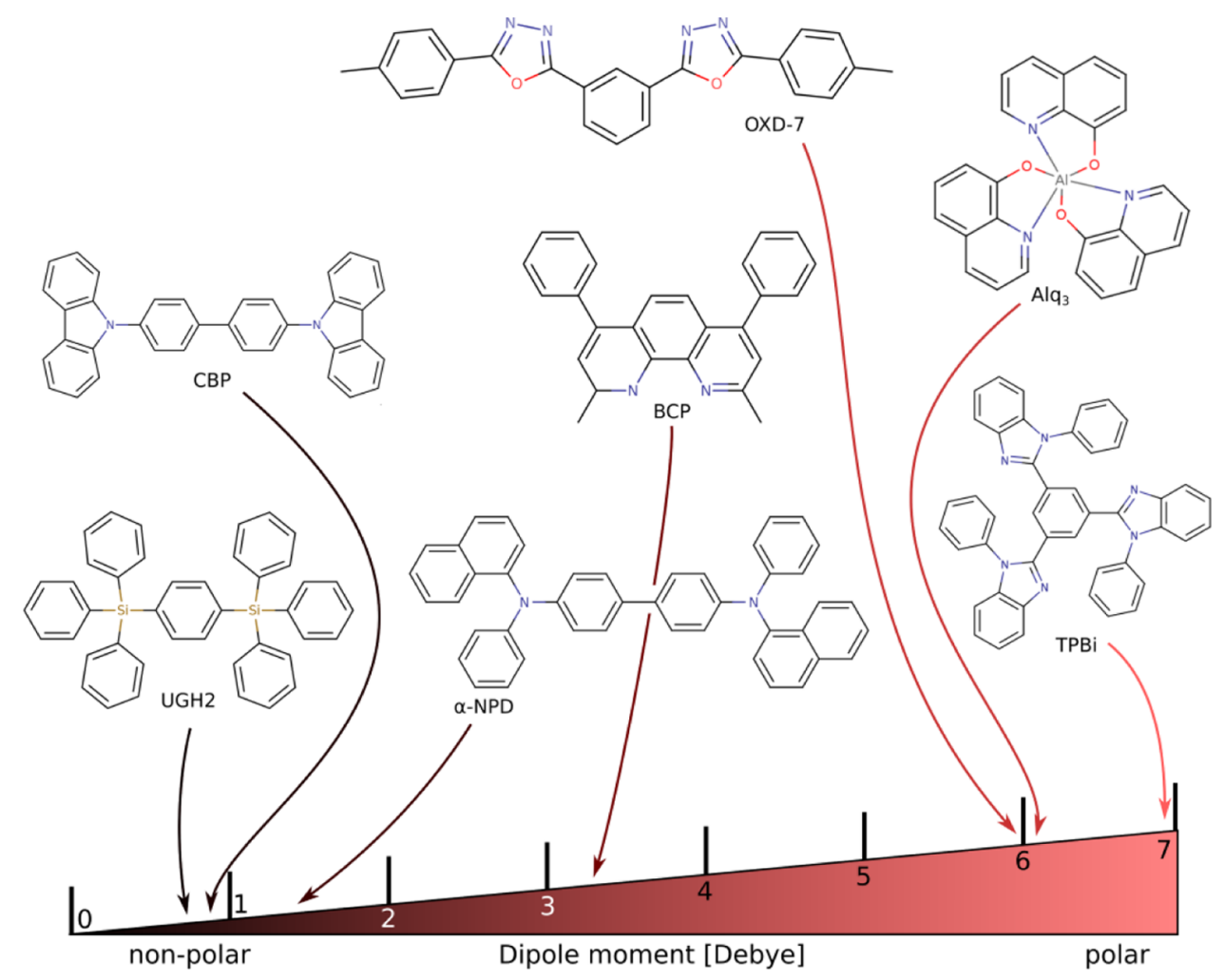

Figure 1. Chemical structures and corresponding dipole moments of all molecules investigated in Noguchi et al. ${ }^{2}$ and in this study.

the anisotropic orientation and evaluate the energy landscape for charge transport in films showing the GSP effect.

\section{SIMULATION METHODS}

Amorphous atomistic morphologies of Tris(8 hydroxyquinolinato) aluminum $\left(\mathrm{Alq}_{3}\right), 4,4^{\prime}$ bis[ $N$ (1 naphthyl) $N$ phenylamino] biphenyl $(\alpha$ NPD), $1,3,5$ tris $(1$ phenyl $1 H$ benzimidazol 2 yl)benzene (TPBi), 2,9 dimethyl 4,7 diphenyl 1,10 phenanthroline (BCP), 1,3 bis[2 (4 tert butylphenyl) 1,3,4 oxadiazo 5 yl] benzene (OXD 7), 1,4 bis (triphenylsilyl)benzene (UGH2), and $4,4^{\prime}$ bis ( $N$ carbazolyl) $1,1^{\prime}$ biphenyl (CBP) (see Figure 1) are generated using a classical force field based Monte Carlo (MC) method. ${ }^{19}$ The method mimics a physical vapor deposition protocol by depositing one molecule at a time. Using a simulated annealing (SA) protocol, the molecules explore the energy landscape on the surface of the already deposited and fixed molecules. We use 10 simulated annealing (SA) cycles with 150000 Monte Carlo steps in each cycle. In each SA cycle, the temperature is decreased from artificially high temperature $(4000 \mathrm{~K})$ to room temperature $(300 \mathrm{~K})$. The end state of each SA cycle is accepted or rejected using the Metropolis Monte Carlo criterion (see basin hopping approaches ${ }^{20}$ ). For each of the materials shown in Figure 1, we generate five independent simulation boxes with 1200 molecules each to obtain representative atomistic models of the amorphous thin films. The deposition simulation starts with an empty simulation box, in which molecules are added at a random position on the $z=z_{0}$ plane of the box, which ranges from $z=-z_{0}$ to $z=z_{0}$. To prepare an initial layer of molecules, we use an additional potential, which forces the molecules to move toward the bottom of the box $(z=$ $-z_{0}$ plane). This potential only influences the $z$ position of the center of mass of the molecule and not the orientation of the molecule. As soon as the first layer of molecules is created, the additional potential is switched off. The first layer of molecules is removed after preparation of the film. The high degree of orientation disorder even in films with high GSP effect as well as the comparatively small number of molecules in the first layer makes it impossible that the additional potential has a systematic impact on the growth of the overall film. Molecular structures and ESP charges ${ }^{21}$ for the parameterization of molecule specific force fields are obtained using density functional theory (DFT) calculations using the def2 SV(P) basis set ${ }^{22}$ and a B3 LYP functional, ${ }^{23}$ as implemented in Turbomole. ${ }^{24}$

To analyze the electronic properties, such as distributions of dipole moments and energy levels in the morphologies, we use the Quantum Patch method. ${ }^{25,26}$ In this method, the electronic structure of all molecules is calculated using self consistently coupled DFT calcu lations. The electrostatic interaction of the weakly coupled molecules is modeled in an iterative procedure to account for interaction effects, such as polarization due to the electrostatic potential of the molecules.

\section{ORIENTATION ANISOTROPY AND GSP EFFECT}

We analyzed the atomistic morphologies in terms of their geometrical and electronic properties. An overview over the geometrical properties is shown in Table 1 . The average volume per molecule ranges from $0.52 \mathrm{~nm}^{3}$ for the smallest molecule (BCP) to $1.01 \mathrm{~nm}^{3}$ for the largest (UGH2) molecule (see Supporting Information (SI)). The molecular dipole moments $\vec{d}$ in the solid state are calculated using the Quantum Patch

Table 1. Analysis of the Atomistic Amorphous Morphologies

$\begin{array}{llccc}\text { system } & \begin{array}{c}\text { number of } \\ \text { molecules }\end{array} & \begin{array}{c}\text { average volume } \\ \text { per molecule } \\ \left(\mathrm{nm}^{3}\right)\end{array} & \begin{array}{c}\text { mean dipole } \\ \text { moment } \\ (\mathrm{D})\end{array} & \begin{array}{c}\text { mean } \\ \text { orientation } \\ \langle o\rangle(\%)\end{array} \\ \mathrm{UGH} 2 & 5 \times 1000 & 1.01 \pm 0.01 & 0.7 \pm 0.3 & 2.7 \pm 0.7 \\ \alpha \text {-NPD } & 5 \times 1000 & 0.87 \pm 0.01 & 1.6 \pm 0.7 & 1.1 \pm 0.8 \\ \mathrm{BCP} & 5 \times 1000 & 0.52 \pm 0.01 & 3.3 \pm 0.6 & 1.6 \pm 0.9 \\ \mathrm{CBP} & 5 \times 1000 & 0.71 \pm 0.01 & 0.9 \pm 0.4 & 8.5 \pm 0.9 \\ \mathrm{TPBi} & 5 \times 1000 & 0.96 \pm 0.01 & 7.0 \pm 3.4 & 5.2 \pm 3.4 \\ \mathrm{Alq} & 5 \times 1000 & 0.61 \pm 0.01 & 6.0 \pm 0.9 & 5.7 \pm 1.4 \\ \mathrm{OXD}_{3} 7 & 5 \times 1000 & 0.53 \pm 0.01 & 6.0 \pm 1.4 & 12.4 \pm 1.7 \\ \mathrm{Alq}_{3} & 3 \times 10000 & 0.62 \pm 0.00 & 5.7 \pm 0.9 & 5.5 \pm 1.4 \\ \mathrm{Alq}_{3} \text { excl. } & 2 \times 10000 & 0.62 \pm 0.00 & 5.1 \pm 1.0 & 10.4 \pm 0.3 \\ \mathrm{ES} & & & & \end{array}$


(a)

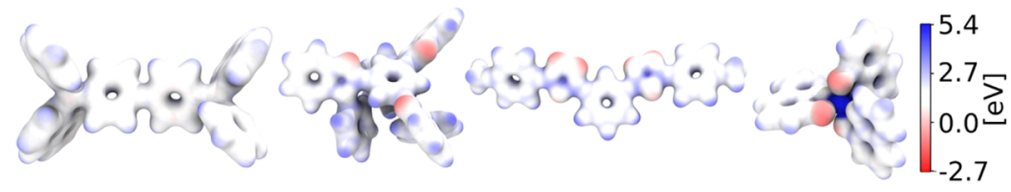

(b)

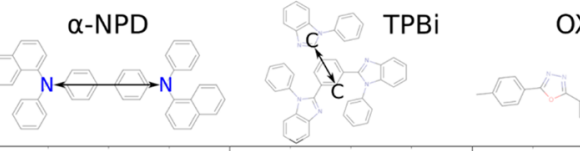

OXD-7

(c)
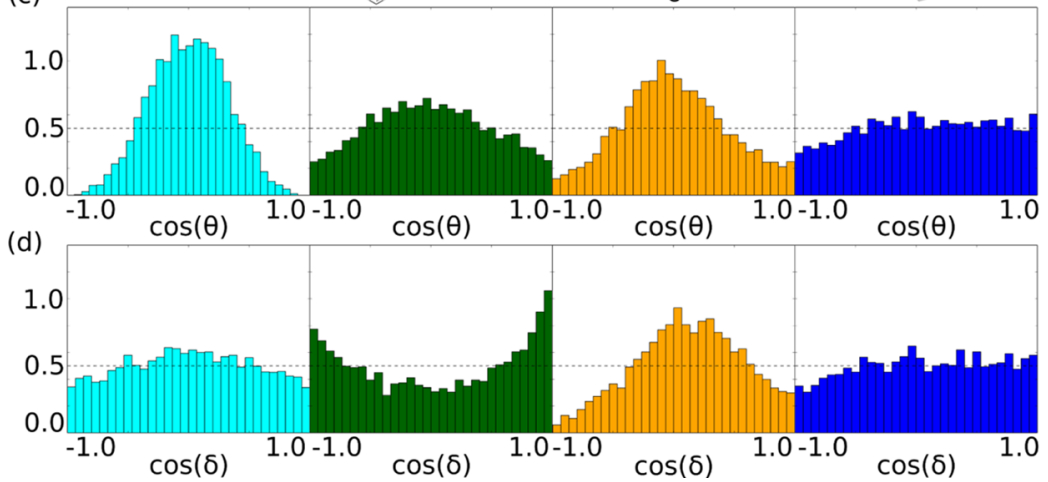

Figure 2. (a) Electrostatic potential of the nonpolar molecule $\alpha$ NPD and the polar molecules TPBi, OXD 7, and Alq $\mathrm{q}_{3}$ on surfaces of constant electron density. (b) Definition of the intramolecular axes used to determine the degree of anisotropy. (c) Distribution of $\cos (\theta)$, with $\theta$ being the angle between the axis indicated in (b) and the growth direction. (d) Distribution of $\cos (\delta)$, with $\delta$ being the angle between the molecular dipole moment and the growth direction. Isotropic angle distributions are indicated by the dashed lines as a reference. The data for UGH2, $\mathrm{BCP}$, and $\mathrm{CBP}$ are shown in Figure S1 in the SI.

method, ${ }^{25,26}$ which accounts for mutual polarization effects between molecules. The electrostatic potentials of the nonpolar molecule $\alpha$ NPD and the polar molecules TPBi, OXD 7, and $\mathrm{Alq}_{3}$ on surfaces of constant electron density are shown in Figure 2a. The figure shows that mainly oxygen and nitrogen atoms in oxydiazole ( $\mathrm{OXD} 7)$, hydroxyquinoline $\left(\mathrm{Alq}_{3}\right)$, and benzimidazole groups (TPBi) generate the polarity, whereas aromatic carbon systems are mostly nonpolar. The mean orientation in growth direction ( $z$ direction) $\langle o\rangle$ of $N$ molecules is calculated as $\langle o\rangle=1 / N \sum_{i=1}^{N}\left|\vec{d}_{i} \cdot \hat{z}\right| /\left|\vec{d}_{i}\right|$. This anisotropy in molecular orientation is depicted in Figure $2 \mathrm{c}$. In agreement with the $\langle o\rangle$ values, significant differences between the observed angle distributions and angle distribu tions of perfectly isotropic morphologies are visible. In case of $\alpha \mathrm{NPD}, \mathrm{TPBi}$, and OXD 7, the molecules tend to lie flat on the surface, which is indicated by a maximum of the probability distribution of $\cos \theta \sim 0$, where $\theta$ is the angle between the molecular axis indicated in Figure $2 \mathrm{~b}$ and the growth direction $z$. As a consequence of this anisotropy in molecular orientation, the dipole moments of the molecules also become oriented in preferred directions. In case of $\alpha \mathrm{NPD}$, the distributions of $\cos \theta$ and $\cos \delta$, with $\delta$ being the angle between the molecular dipole moment and growth direction, are almost symmetric. In contrast, the molecular axis and dipole moment of TPBi and OXD 7 are slightly more often oriented in $+\hat{z}$ direction compared to $-\hat{z}$ direction. This leads to a net orientation of the molecular dipole moments and therefore to a net dipole moment of the thin film. In case of $\mathrm{Alq}_{3}$, the molecular dipole moment nearly coincides with the $\mathrm{N}-\mathrm{Al}-\mathrm{O}$ axis of the molecule. Thus, the distributions of $\cos \theta$ and $\cos \delta$ are very similar. For $\mathrm{Alq}_{3}$, we also observe a suppression of the orientation effect in $-\hat{z}$ direction, leading to a net orientation and to the GSP effect.
To understand the cause of the observed ordering effect, we additionally generated larger $\mathrm{Alq}_{3}$ morphologies with and without taking electrostatic interaction into account. In these artificial simulations, intermolecular interaction is modeled using only classical Lennard Jones potentials representing short range Pauli repulsion and medium range van der Waals interaction. As shown in the bottom two lines of Table 1 and in Table S1 in the SI, the GSP effect becomes even stronger in the absence of electrostatic (mainly dipole-dipole) interaction during the deposition. This suggests that intermolecular electrostatic interaction weakens the maximum possible molecular ordering by favoring molecular orientations, which reduce the GSP. We therefore conclude that the cause of the GSP effect is related to short range interaction between the molecules and the film. This is in agreement with the results reported by Jurow et al., ${ }^{9}$ where the orientation effect was suspected to be caused by the intrinsically distorted symmetry at the interface between organic material and vacuum.

On the basis of the data given in Table 1, the strength of the GSP can be estimated. If we assume an infinite layer with finite thickness $t$, which is filled with molecules with average volume $V$, dipole moment $d$, and a mean orientation $\langle o\rangle$, then the GSP can be estimated as

$$
\mathrm{GSP}=\frac{1}{\varepsilon \varepsilon_{0}} \frac{d\langle o\rangle}{V} t \equiv \alpha t
$$

The slope $\alpha$ of the GSP therefore only depends on the quantities given in Table 1

$$
\alpha=\frac{1}{\varepsilon \varepsilon_{0}} \frac{d\langle o\rangle}{V}
$$

We assume the relative permittivity to be $\varepsilon=3.5 \pm 0.5$ for all molecules. 
The simulation results as well as experimental data from Ishii et al. are shown in Figure $3 .^{2}$ The theoretical findings reproduce the trends and order of magnitude of the GSP effect observed in experiment.

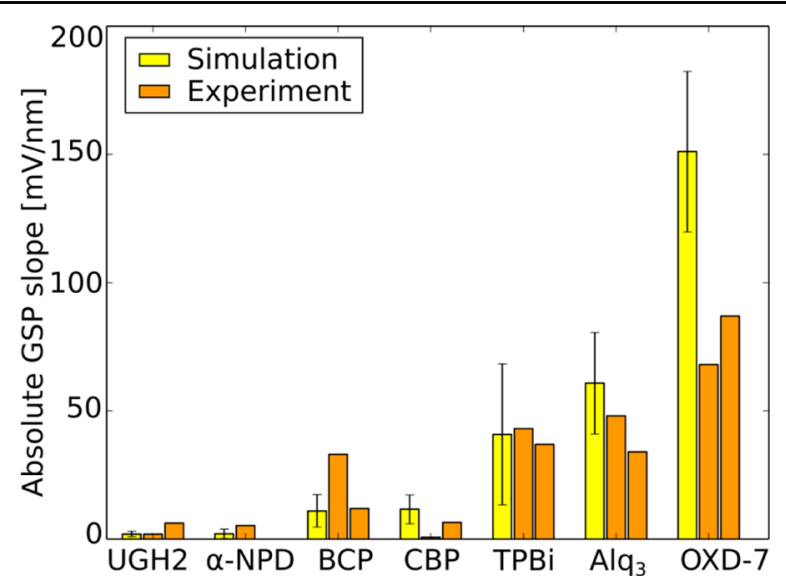

Figure 3. Comparison of the calculated and the measured GSP strengths. For all molecules except OXD 7, the values calculated using eq 2 agree almost quantitatively with the experimental data found in Ishii et al. ${ }^{2}$ The two experimental bars are measured using the Kelvin probe (KP) method and the DCM method ${ }^{27,28}$ using the FCE 1 measurement system for ferroelectrics. Deviations between experiment and simulation, particularly in case of $\operatorname{OXD} 7$, might arise from approximations made to describe intermolecular interactions in a classical force field.

\section{DENSITY OF STATES AND ENERGY DISORDER}

The GSP effect investigated in the last section directly effects electron and hole transport through the formation of a built in electrostatic field. Charge transport in amorphous semi conductors is described by the theory of hopping transport, where localized charge carriers move through the disordered system in a sequence of hopping processes. The charge hopping rate can be estimated using Marcus theory, ${ }^{29}$ and it exponentially depends on site energy differences and thus the local energy landscape. ${ }^{30}$ Many models describe the charge carrier mobility as a function of the energy disorder $\sigma,^{30-35}$ which is usually defined as the width of the global density of states. It was found that models assuming a purely Gaussian density of states fail in many cases, which led to the development of models, including correlated disorder. ${ }^{32,34}$ Although such correlations mainly arise from the electrostatic interaction between molecules, the anisotropic and partially ordered orientation of molecules described in this work also leads to correlations in the energy levels and influence charge transport. To analyze the influence of the GSP effect on the distribution of energy levels in the amorphous thin films, we extracted the molecular orbital energies of the amorphous systems using the Quantum Patch approach. ${ }^{25,26}$ In this approach, a system of weakly interacting molecules is decoupled and self consistently analyzed using an iterative scheme of single molecule DFT calculations. In each calculation, the respective molecule is surrounded by an iteratively updated cloud of point charges of all other molecules representing the electrostatic background of the surrounding molecules.

The spatially resolved lowest unoccupied molecular orbital (LUMO) energies of one of the $\mathrm{Alq}_{3}$ systems are shown in Figure 4a. We observe a strong gradient of the energies in $z$ direction. Specifically, highest occupied molecular orbital (HOMO) and LUMO energies as well as the electrostatic potential caused by all point charges in the system linearly decrease as a function of the $z$ coordinate (see Figure $4 \mathrm{~b}$ ). This suggests that the averaged electrostatic potential generated by the partially ordered molecules in the thin film is responsible for the shift of the orbital energies and the GSP effect discussed in the previous section. In addition to the long range electrostatic potential causing the GSP effect, short range interactions cause a local distribution of energy levels. The width of this local energy distribution, the energy disorder $\sigma$, exponentially influences the charge carrier mobility. ${ }^{36,37}$

Similarly to other materials with correlated disorder, ${ }^{32,38}$ it is necessary to differentiate between global disorder, as shown in Figure 5a, and local disorder, which can be estimated by the analysis of site energy differences of pairs with a center of mass distance within a certain cutoff. The deviation between local and global disorder is a measure of spatial correlations of the site energies and therefore influences the charge carrier mobility. It thus needs to be considered in all types of charge carrier mobility calculations using numerical kinetic (a)

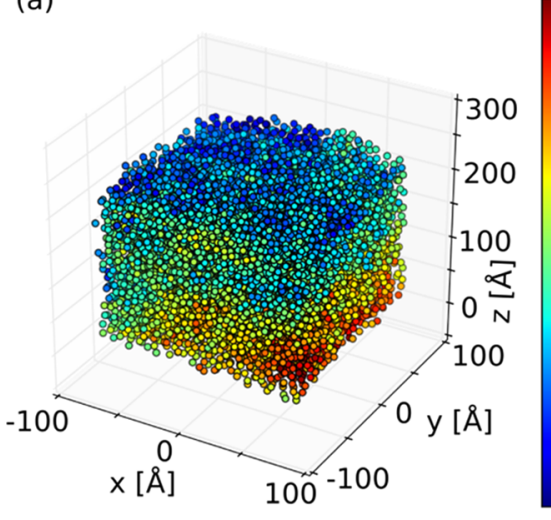

(b)

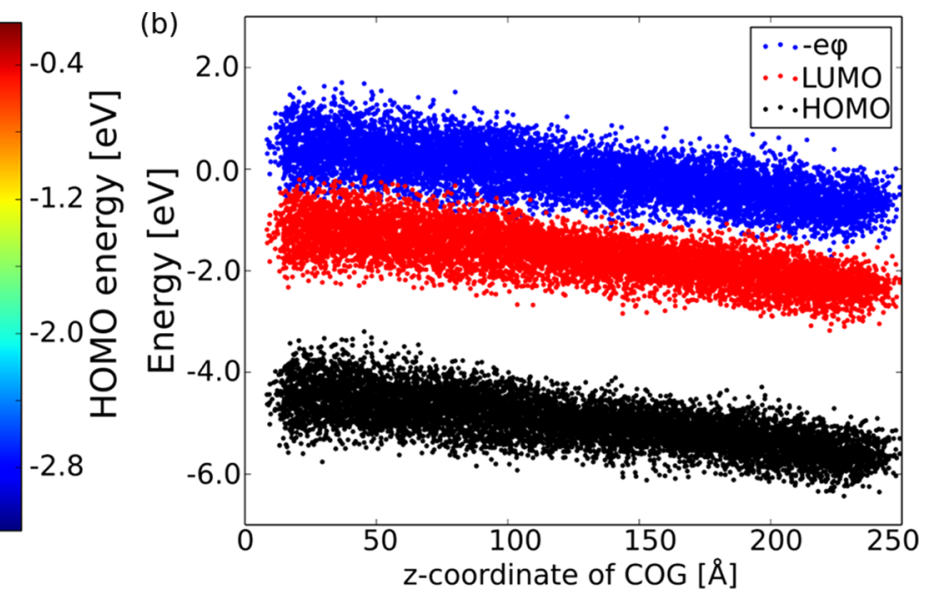

Figure 4. (a) Scatter plot of LUMO energies and (b) the electrostatic potential in the $\mathrm{Alq}_{3}$ system at the center of mass positions of the respective molecules. An energy gradient in $z$ direction is visible, indicating a built in electric field caused by the dipole moments of the partially oriented Alq $q_{3}$ molecules. 


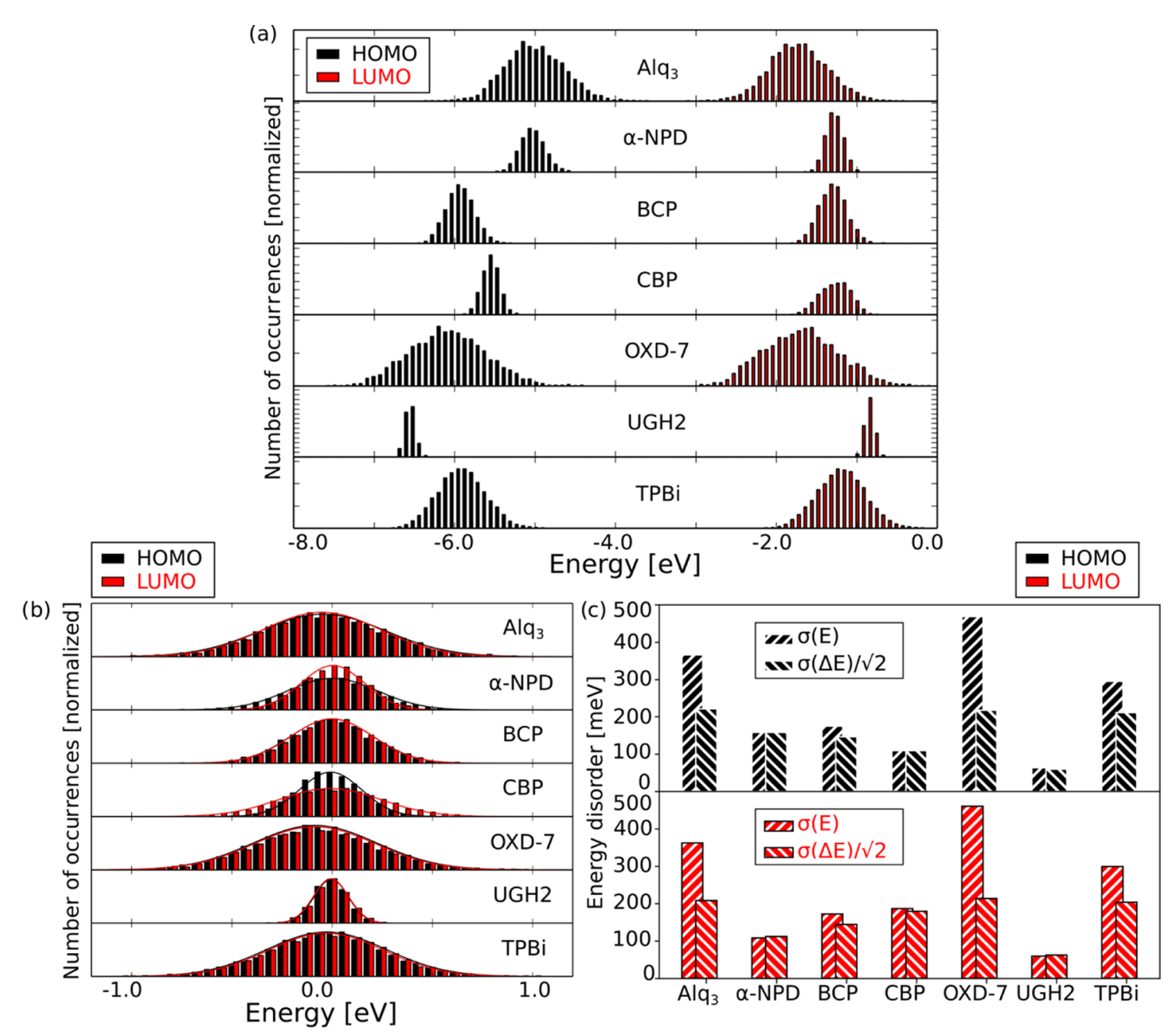

Figure 5. (a) Global distribution of HOMO and LUMO energies and (b) local distribution of HOMO and LUMO energy differences for pairs with up to $\sim 20 \AA$ center of mass distance. Due to the GSP effect, the energy levels shown in (a) do not follow a Gaussian distribution. In addition to that, their distribution is much broader than expected from the local energy differences as shown in (b). (c) Direct comparison between local $(\sigma(\Delta E) /$ $\sqrt{ } 2$ ) and global energy disorder $(\sigma(E))$ (standard deviation of the Gaussian distribution of energy levels). The deviation is strongest in case of a strong GSP effect $\left(\mathrm{Alq}_{3}, \mathrm{OXD} 7\right.$, and TPBi).

Monte Carlo simulations or analytical models, such as the Gaussian disorder model.

The distribution of site energy differences with a pair cutoff distance of $20 \AA$ is Gaussian, as shown in Figure 5b. In uncorrelated systems with a global Gaussian disorder, the width of the global DOS and the width of the distribution of local site energy differences have a ratio of $1 / \sqrt{2}$. In the presence of the GSP effect (mainly in Alq3, OXD 7, and TPBi), we observe a deviation between the global disorder $\sigma(E)$ and the local energy disorder $\sigma(\Delta E) / \sqrt{ } 2$ (see Figure 5c), which can be attributed to spatial correlations due to the ordering of the dipole moments.

\section{SUMMARY}

In summary, we have demonstrated that a molecular simulation protocol mimicking physical vapor deposition can efficiently simulate the emergence of partially ordered morphologies as a consequence of the broken symmetry between the deposited film and the vacuum region, an effect not observed in bulk simulations. For molecules with a dipole moment, this partial ordering leads to the formation of a giant surface potential in agreement with experimental observations, but the dipole moment is not directly responsible for the ordering, instead the local nonbonded interactions of the molecule with the surface are the driving force for the observed ordering effect. This finding can potentially be used for systematic modifications of molecules to control the GSP effect or more generally the orientation of molecules in amorphous thin films.

By coupling the morphology simulation with an analysis of the density of states, we find that the mobility in the material is affected by the GSP effect. To properly estimate the mobility, the local density of states, which is corrected for the shift of the center of the distribution as a function of the position in the film, must be used. We find that large carrier mobility and a strong GSP effect are hard to realize at the same time. A large intrinsic dipole moment is required for the GSP effect, and random distributions of large dipole moments create a rough energy landscape and strong (local) energy disorder, which reduces the carrier mobility and conductivity of a material.

\section{ASSOCIATED CONTENT}

\section{Supporting Information}

The Supporting Information is available free of charge on the ACS Publications website at DOI: 10.1021 /acsami.7b11762. 
Calculation of the molecular volumes, mean orientation with and without electrostatic interaction, orientation distributions (PDF)

\section{AUTHOR INFORMATION}

\section{Corresponding Authors}

*E mail: florian.vonwrochem@sony.com (F.v.W.).

*E mail: wolfgang.wenzel@kit.edu (W.W.).

\section{ORCID}

Pascal Friederich: 0000000344651465

Florian von Wrochem: 0000000322989270

\section{Notes}

The authors declare no competing financial interest.

\section{ACKNOWLEDGMENTS}

The authors thank S. Hattori, M. Nakamoto, and Y. Tokita for fruitful discussions. Furthermore, they acknowledge funding by the Helmholtz program "Science and Technology of Nano systems" (STN) and the H2020 project EXTMOS (grant number: 646176). This work was performed on the computa tional resource ForHLR Phase I funded by the Ministry of Science, Research and the Arts Baden Württemberg and DFG ("Deutsche Forschungsgemeinschaft"). P.F. thanks the Carl Zeiss Foundation for funding the project "Multiskalen Modellierung elektronischer Eigenschaften von Materialien in der organischen Elektronik".

\section{REFERENCES}

(1) Berleb, S.; Brütting, W.; Paasch, G. Interfacial charges and electric field distribution in organic hetero layer light emitting devices. Org. Electron. 2000, 1, 41-47.

(2) Noguchi, Y.; Miyazaki, Y.; Tanaka, Y.; Sato, N.; Nakayama, Y.; Schmidt, T. D.; Brütting, W.; Ishii, H. Charge accumulation at organic semiconductor interfaces due to a permanent dipole moment and its orientational order in bilayer devices. J. Appl. Phys. 2012, 111, No. 114508 .

(3) Ito, E.; Washizu, Y.; Hayashi, N.; Ishii, H.; Matsuie, N.; Tsuboi, K.; Ouchi, Y.; Harima, Y.; Yamashita, K.; Seki, K. Spontaneous buildup of giant surface potential by vacuum deposition of Alq 3 and its removal by visible light irradiation. J. Appl. Phys. 2002, 92, 7306-7310.

(4) Lampe, T.; Schmidt, T. D.; Jurow, M. J.; Djurovich, P. I.; Thompson, M. E.; Brütting, W. Dependence of phosphorescent emitter orientation on deposition technique in doped organic films. Chem. Mater. 2016, 28, 712-715.

(5) Noguchi, Y.; Sato, N.; Miyazaki, Y.; Ishii, H. Light and ion gauge induced space charges in tris ( 8 hydroxyquinolate) aluminum based organic light emitting diodes. Appl. Phys. Lett. 2010, 96, No. 143305.

(6) Campbell, I. H.; Rubin, S.; Zawodzinski, T.; Kress, J.; Martin, R.; Smith, D.; Barashkov, N.; Ferraris, J. Controlling Schottky energy barriers in organic electronic devices using self assembled monolayers. Phys. Rev. B 1996, 54, No. R14321.

(7) Heimel, G.; Romaner, L.; Zojer, E.; Bredas, J. L. The interface energetics of self assembled monolayers on metals. Acc. Chem. Res. 2008, 41, 721-729.

(8) Ford, W. E.; Gao, D.; Knorr, N.; Wirtz, R.; Scholz, F.; Karipidou, Z.; Ogasawara, K.; Rosselli, S.; Rodin, V.; Nelles, G.; et al. Organic dipole layers for ultralow work function electrodes. ACS Nano 2014, 8 , 9173-9180.

(9) Jurow, M. J.; Mayr, C.; Schmidt, T. D.; Lampe, T.; Djurovich, P. I.; Brütting, W.; Thompson, M. E. Understanding and predicting the orientation of heteroleptic phosphors in organic light emitting materials. Nat. Mater. 2016, 15, 85-91.

(10) Schmidt, T. D.; Setz, D. S.; Flämmich, M.; Frischeisen, J.; Michaelis, D.; Krummacher, B. C.; Danz, N.; Brütting, W. Evidence for non isotropic emitter orientation in a red phosphorescent organic light emitting diode and its implications for determining the emitter's radiative quantum efficiency. Appl. Phys. Lett. 2011, 99, No. 163302.

(11) Kim, K. H.; Moon, C. K.; Lee, J. H.; Kim, S. Y.; Kim, J. J. Highly Efficient Organic Light Emitting Diodes with Phosphorescent Emitters Having High Quantum Yield and Horizontal Orientation of Transition Dipole Moments. Adv. Mater. 2014, 26, 3844-3847.

(12) Kim, S. Y.; Jeong, W. I.; Mayr, C.; Park, Y. S.; Kim, K. H.; Lee, J. H.; Moon, C. K.; Brütting, W.; Kim, J. J. Organic Light Emitting diodes with $30 \%$ external quantum efficiency based on a horizontally oriented emitter. Adv. Funct. Mater. 2013, 23, 3896-3900.

(13) Liehm, P.; Murawski, C.; Furno, M.; Lüssem, B.; Leo, K.; Gather, M. C. Comparing the emissive dipole orientation of two similar phosphorescent green emitter molecules in highly efficient organic light emitting diodes. Appl. Phys. Lett. 2012, 101, No. 253304.

(14) Kim, K. H.; Lee, S.; Moon, C. K.; Kim, S. Y.; Park, Y. S.; Lee, J. H.; Lee, J. W.; Huh, J.; You, Y.; Kim, J. J. Phosphorescent dye based supramolecules for high efficiency organic light emitting diodes. Nat. Commun. 2014, 5, No. 4769.

(15) Graf, A.; Liehm, P.; Murawski, C.; Hofmann, S.; Leo, K.; Gather, M. C. Correlating the transition dipole moment orientation of phosphorescent emitter molecules in OLEDs with basic material properties. J. Mater. Chem. C 2014, 2, 10298-10304.

(16) Reineke, S.; Rosenow, T. C.; Lüssem, B.; Leo, K. Improved High Brightness Efficiency of Phosphorescent Organic LEDs Com prising Emitter Molecules with Small Permanent Dipole Moments. Adv. Mater. 2010, 22, 3189-3193.

(17) Kim, K. H.; Ma, J. Y.; Moon, C. K.; Lee, J. H.; Baek, J. Y.; Kim, Y. H.; Kim, J. J. Controlling Emitting Dipole Orientation with Methyl Substituents on Main Ligand of Iridium Complexes for Highly Efficient Phosphorescent Organic Light Emitting Diodes. Adv. Opt. Mater. 2015, 3, 1191-1196.

(18) Moon, C. K.; Kim, K. H.; Lee, J. W.; Kim, J. J. Influence of host molecules on emitting dipole orientation of phosphorescent iridium complexes. Chem. Mater. 2015, 27, 2767-2769.

(19) Neumann, T.; Danilov, D.; Lennartz, C.; Wenzel, W. Modeling disordered morphologies in organic semiconductors. J. Comput. Chem. 2013, 34, 2716-2725.

(20) Rondina, G. G.; Da Silva, J. L. Revised basin hopping Monte Carlo algorithm for structure optimization of clusters and nano particles. J. Chem. Inf. Model. 2013, 53, 2282-2298.

(21) Besler, B. H.; Merz, K. M.; Kollman, P. A. Atomic charges derived from semiempirical methods. J. Comput. Chem. 1990, 11, 431439.

(22) Schafer, A.; Horn, H.; Ahlrichs, R. Fully optimized contracted Gaussian basis sets for atoms Li to Kr. J. Chem. Phys. 1992, 97, 25712577.

(23) Becke, A. D. A new mixing of Hartree Fock and local density functional theories. J. Chem. Phys. 1993, 98, 1372-1377.

(24) Ahlrichs, R.; Bär, M.; Häser, M.; Horn, H.; Kölmel, C. Electronic structure calculations on workstation computers: The program system turbomole. Chem. Phys. Lett. 1989, 162, 165-169.

(25) Friederich, P.; Symalla, F.; Meded, V.; Neumann, T.; Wenzel, W. Ab Initio Treatment of Disorder Effects in Amorphous Organic Materials: Toward Parameter Free Materials Simulation. J. Chem. Theory Comput. 2014, 10, 3720-3725.

(26) Friederich, P.; Meded, V.; Symalla, F.; Elstner, M.; Wenzel, W. A QM/QM approach to model energy disorder in amorphous organic semiconductors. J. Chem. Theory Comput. 2015, 11, 560-567.

(27) Egusa, S.; Miura, A.; Gemma, N.; Azuma, M. Carrier injection characteristics of organic electroluminescent devices. Jpn. J. Appl. Phys. 1994, 33, 2741.

(28) Ogawa, S.; Kimura, Y.; Ishii, H.; Niwano, M. Carrier injection characteristics in organic field effect transistors studied by displace ment current measurement. Jpn. J. Appl. Phys. 2003, 42, No. L1275.

(29) Marcus, R. A. Chemical and Electrochemical Electron Transfer Theory. Annu. Rev. Phys. Chem. 1964, 15, 155-196.

(30) Bässler, H. Localized states and electronic transport in single component organic solids with diagonal disorder. Phys. Status Solidi B 1981, 107, 9-54. 
(31) Borsenberger, P.; Bässler, H. Concerning the role of dipolar disorder on charge transport in molecularly doped polymers. J. Chem. Phys. 1991, 95, 5327-5331.

(32) Van Mensfoort, S. L. M.; Shabro, V.; de Vries, R. J.; Janssen, R. A. J.; Coehoorn, R. Hole transport in the organic small molecule material $\alpha$ NPD: evidence for the presence of correlated disorder. J. Appl. Phys. 2010, 107, No. 113710.

(33) Coehoorn, R.; van Mensfoort, S. L. M. Effects of disorder on the current density and recombination profile in organic light emitting diodes. Phys. Rev. B 2009, 80, No. 085302.

(34) Massé, A.; Friederich, P.; Symalla, F.; Liu, F.; Meded, V.; Coehoorn, R.; Wenzel, W.; Bobbert, P. A. Effects of energy correlations and superexchange on charge transport and exciton formation in amorphous molecular semiconductors: An ab initio study. Phys. Rev. B 2017, 95, No. 115204.

(35) Fishchuk, I.; Kadashchuk, A.; Hoffmann, S.; Athanasopoulos, S.; Genoe, J.; Bässler, H.; Köhler, A. Unified description for hopping transport in organic semiconductors including both energetic disorder and polaronic contributions. Phys. Rev. B 2013, 88, No. 125202.

(36) Rodin, V.; Symalla, F.; Friederich, P.; Meded, V.; Danilov, D.; Poschlad, A.; Nelles, G.; von Wrochem, F.; Wenzel, W. A generalized effective medium model for the carrier mobility in amorphous organic semiconducturs. Phys. Rev. B 2015, 91, No. 155203.

(37) Friederich, P.; Meded, V.; Poschlad, A.; Neumann, T.; Rodin, V.; Stehr, V.; Symalla, F.; Danilov, D.; Lüdemann, G.; Fink, R.; Kondov, I.; von Wrochem, F.; Wenzel, W. Molecular origin of the charge carrier mobility in small molecule organic semiconductors. $A d v$. Funct. Mater. 2016, 26, 5757-5763.

(38) Massé, A.; Friederich, P.; Liu, F.; Nitsche, R.; Coehoorn, R.; Wenzel, W.; Bobbert, P. A.; et al. Ab initio charge carrier mobility model of amorphous molecular semiconductors. Phys. Rev. B 2016, 93, No. 195209. 
Karlsruher Institut für Technologie

\section{Repository KITopen}

Dies ist ein Postprint/begutachtetes Manuskript.

Empfohlene Zitierung:

Friederich, P.; Rodin, V.; Wrochem, F. von; Wenzel, W.

Built-In Potentials Induced by Molecular Order in Amorphous Organic Thin Films.

2018. ACS applied materials \& interfaces, 10

doi:10.5445/IR/1000080307

Zitierung der Originalveröffentlichung:

Friederich, P.; Rodin, V.; Wrochem, F. von; Wenzel, W.

Built-In Potentials Induced by Molecular Order in Amorphous Organic Thin Films.

2018. ACS applied materials \& interfaces, 10 (2), 1881-1887.

doi:10.1021/acsami.7b11762

Lizenzinformationen: KITopen-Lizenz 